\title{
LA CIENCIA ECONOMICA Y EL ANÁLISIS ENERGÉTICO. DISCUSIONES ANTIGUAS Y RECIENTES
}

\author{
J. Martínez Alier \\ (Universidad Autónoma de Barcelona)
}

\section{ECONOMAA, ECOLOGIA Y CREMATISTICA}

Que muchos de los conceptos de la teoría económica son ajenos a la realidad de la naturaleza es hoy cosa muy aceptada. Por ejemplo, en los textos de economía uno encuentra todavía menciones a la «producción» de petróleo de tal o cual país, refiriéndose no por supuesto a la producción sino a la extracción y eventual destrucción. Por ejemplo, la teoría econó. mica sería incapaz de explicar por qué es imposible, cualquiera que fuera el nivel de ingresos, que la densidad y el uso de automóviles ptivados de gasolina en el mundo sea similar al de California o aun al de Cataluña.

Cuando se comprueba que muchos de los conceptos de la ciencia económica son de catácter metafísico, existe la tentación de pensar que el estudio de la economía es superftuo. $\mathrm{Si}$, para una especie animal, la «ecología» estudia cómo utiliza el flujo de energía y de materiales, y esa «ecología» es su «economía», ¿por qué ha de ser distinto con la humanidad?

Sin embargo, el núcleo de la ciencia económica que es el estudio de cómo se asignan los recursos entre distintas actividades (en un momento dado y en el tiempo), y cómo se teparten entre la población los frutos de esas actividades, no puede reducirse, para la especie humana, a la «ecolo. gía». Tratar de entender la historia económica de la humanidad o de distintos segmentos de la humanidad a la luz de su adaptacion a la disponibilidad de recursos energéticos y materiales, dejaría de lado buena parte de esa historia, como por ejemplo el muy distinto camino seguido por el 
Japón e Indonesia. Es tal vez, precisamente, la falta de adaptación a las disponibilidades continuadas de energía y de materias primas, es el uso excluyente de unos stocks agotables de energía fósil y de materias primas por parte de algunos países y de algunos sectotes dentro de algunos países (uso excluyente inicial, que permite adquirir el poder para continuarlo después) lo que puede ayudar a explicar la reciente historia económica mundial. Creo que en ninguna especie animal existen diferencias en el consumo de energía y de materiales como las que existen entre un ciudadano tico de Los Angeles y un ciudadano pobre de Calcuta. Los procesos y decisiones que han llevado a esa situación (más los procesos y decisiones que pueden cambiarla) son campo de estudio de las ciencias sociales, de las ciencias de la "cultura» (no son cuestiones físicas, químicas, genéticas).

La economía estudia cómo se asignan los recursos entre distintas actividades. Este tipo de estudio debería seguir un camino intermedio entre la ecología y la «crematística», siendo esta última el estudio de la formación de los precios. Aunque a primera vista parezca que la importancia relativa de las distintas actividades económicas venga determinada por los precios, un segundo análisis revela que depende de la distribución del poder de compra. Ese poder de compra depende de cuestiones extra-econónicas, es decit, to se determina exclusivamente en el mexcado de los servicios de los «factores de la producción», sino que influyen en gran manera la historia de la distribución de la propiedad en la sociedad de que se trate, la historia de las oportunidades educativas, la jerarquía del prestigio de ocupaciones y las sanciones sociales que existen para reforzar (eventualmente, para menoscabar) esa jerarquía, las costumbres que afecten a la oferta de trabajo (femenino o infantil, por ejemplo), las costumbres que determinen la propiedad y uso (colectivo, individual) de la tierra, etc.

\section{EL CRITERIO DE GRAY.HOTELLING}

Veamos la cuestión del precio de los recursos naturales agotables, que requiere en teoría económica un tratamiento distinto a los dernás precios.

La pauta de extracción de recursos naturales agotables que optimizaría su uso a lo largo del tiernpo (para unas demandas conocidas) se obtendría mediante el empleo de la llamada regla de Gray-Hotelling, tal como se expone a continuación.

Si ahora vendemos un bartil extra de petróleo que reporta, por ejemplo, 2.000 pesetas (diferencia entre el precio de venta y el coste de extrac- 
ción), y si colocamos este dinero a interés (por ejemplo, al 20 por ciento), dentro de un año tendremos, como consecuencia de la venta actual, 2.400 pesetas. Si nuestra expectativa de aumento de precio entre ahora y el año que viene es superior, entonces deciditemos no extraer ni vender ese barril extra, sino conservarlo para venderlo dentro de un año. Es decir, el criterio a seguir por el extractor-vendedot no es el de extraer la cantidad que en un momento dado iguala coste marginal de extracción e ingreso marginal de la venta, sino el criterio de maximizar el valor actual de la corriente de ingresos netos a obtener mientras le dure el stock de recurso escaso agotable.

Esto sucede cuando la diferencia (precio-coste marginal de extracción) crece cada año igual que la tasa de interés. La tasa de interés es lo que permite averiguar el valor actual de un ingreso futuro. Así, si la tasa de interés es el 20 por ciento «descontamos el futuro» de tal manera que da lo mismo 2.400 pesetas el año próximo que 2.000 ahora, da lo mismo 120 pesetas el año próximo que 100 ahora. El valor actual de 120 pesetas de ingresos dentro de un año es 100 pesetas ahora.

Si la tasa de interés fuera cero, eso quertía decir que no se «descuenta el futuro». Normalmente se supone que para una persona es lógico descontar el futuro: podemos preferir 100 pesetas hoy a 105 el año próximo porque hoy estamos vivos y no sabemos si lo estaremos el año próximo.

Otra razón que pudiera hacet preferir un poder de compra inferior boy a un poder de compra algo superior el año próximo podría ser la expectativa de que el año próximo seremos de cualquier modo más ricos: por ejemplo, un heredeto prospectivo podría pedir un préstamo a un banco y pagar un alto interés si espera hetedar pronto. Una peseta de hoy le vale mucho más que una peseta del mañana, porque espera tener muchas pesetas tranana y hoy tiene pocas. Ahora bien: generalizat y admitir esa preferencia subjetiva del presente sobre el futuro (aceptat las preferencias es típico del individualismo metodológico que caracteriza a la teoría económica ortodoxa) tendría sentido si realmente estuviéramos en la feliz circunstancia del beredero potencial.

\section{LAS ADVERTENCIAS DE RUDOLF CLAUSIUS}

Pero los países ricos (y las personas ricas de los países pobres) no tienen seguramente derecho a considerarse como herederos potenciales. Les sería aplicable en todo caso la metáfora de herederos dilapidadores, que se están gastando en juergas una herencia ya recibida. Esta metáfora, tan 
cara a Lewis Mumford, parece que fue por primera vez expuesta en el contexto de una discusion similar a la actual por alguien tan bien cualificado como Rudolf Clausius, quiez en 1885 incluyo las páginas siguientes en una conferencia titulada «Sobre las reservas de energía de la naturaleza y su valorización para uso de la humanidad»:

«Vivimos ahora, con respecto al uso de energía mecánica, en un tiempo maravilloso. En general, en las relaciones económicas, vale el principio de que de cada cosa puede usarse sólo lo que en el mismo tiempo pueda ser de nuevo producida. Por tanto, se debería usar como material combustible sólo la cantidad que es producida de nuevo a través del crecimiento de los árboles. Peto en verdad nos comportamos de manera muy distinta.

»Hemos hallado que hay bajo la tierra teservas de carbón de tiempos antiguos que se han formado de plantas en la superficie de la tierra y depositado masivamente durante un período tan largo que los tiempos históricos, en comparación, parecen minúsculos.

"Los gastamos ahora y nos comportamos exactamente como herederos felices que consumen un rico patrimonio. Se saca de la tierra todo lo que permite la fuerza humana y los medios auxiliares técnicos, y se usa como si fuera inagotable. La cantidad de trenes, barcos de vapor y fábricas con máquinas de vapor que usan carbón aumenta de manera tan sorprendente que, mirando al futuro, no es algo caprichoso el preguntarse qué ocurrirá cuando los yacimientos de carbón queden agotados.

"No puede decirse que esto sea una cuestión supetfiua por la tiqueza de estos yacimientos. En toda la tierra son aún muy grandes, sin que de mo. mento exista una estimación de las reservas, pero para países individuales puede verse ya cuáles son las reservas y cuánto tiempo durarán $(\ldots)$. Cuando se habla de tales eventualidades, se escucha a veces la objeción de que antes de que se agoten los yacimientos de carbón se habrá encontrado desde hace tiempo nuevos medios de producir calor, de manera que no hace falta preocuparse. Si se pregunta, sin embargo, cuáles deben ser estos descubrimientos, aparecen puntos de vista como que tal vez se tendrá éxito en separar el agua en sus partes constituyentes, oxígeno e hidrógeno, sin gasto de energía, y con eso podría abrirse una fuente de calor inagotable mediante el calor de combustión del hidrógeno.

»Esos puntos de vista contradicen, no obstante, de manera total, los principios básicos de la física. No se trata aquí en absoluto de sopesar probabilidades sino que puede distinguirse con total certidumbre lo posible de lo imposible. Cualquier creación de energía sin un gasto correspondiente de energía es absolutamente imposible. La reserva de energía potencial que está disponible en los yacimientos de carbón debe su carácter excep. 
cional a aquella energía enviada por el sol a la tierra en la forma de calor radiante necesario para la nutrición de las plantas, en un período de tiempo más largo que el de existencia de la especie humana. Cuando esta teserva se haya gastado, ningún medio de la ciencia por avanzada que sea estará en situación de abrir otra fuente de energía, sino que los hombres se vetán obligados a arreglárselas con la energía que el sol, todavía continuamente a lo largo de los tiempos futuros, les ofrezca mediante sus rayos.

»Esta enetgía se ofrece, por un lado, en el material capaz de oxidación producido por el crecimiento continuo de las plantas y, por otto lado, en los movimientos del tipo del viento y del agua que cae y que corre, y que igualmente se deben al efecto de los rayos solares. Especialmente los movimientos del agua pueden proporcionar tanta energía útil, que una gran caída de agua puede sustituir una mina de carbón de considetable tamaño y de manera semejante se comportan todos los ríos, corrientes y arroyos que corren tápidamente. Hasta ahora esta energía del agua en movimiento es utilizada por los molinos sólo en medida muy reducida. Desperdiciamos la mayor parte de esta energía. De hecho, en el caso de una caída de agua de tamaño considerable o de un río con fuerte corriente es muy difícil utilizar toda la energía disponible en el mismo sitio de forma eficaz. Pero si fuese posible transportar esta energía a otros sitios distantes, entonces sería bastante más fácil su utilización eficaz. Para hacer esto, tenemos ahora la máquina dinamoeléctrica y pienso que en esto consistirá su utilización principal en el futuro (...). Para uta amigo de la naturaleza, por supuesto, no puede ser una imagen atractiva el pensar que las cataratas que ahora con su bravura espumarte forman un adorno principal de las montañas, sean capturadas y enganchadas a máquinas. Pero este destino no puede evitarse $(\ldots)$.

»Mientras el siglo pasado se ha distinguido por la invención o perfeccionamiento de máquinas, entre las cuales destaca la máquina de vapor, que pone al servicio del hombre las fuerzas de la naturaleza de una maneta antes nunca sospechada, ahora los siglos siguientes tendrán la tarea de introducir una sabia econonía en el gasto de las fuentes de fuerza de la natutaleza que se nos ofrecen y especialmente no desperdiciar de manera derrochadora aquelia que encontramos en la tierta como herencia de épocas pasadas y que no se puede sustituir por nada. Cuanto antes llegue un cambio tanto mejor será para el futuro. Las naciones que están en la cima de la civilización tendrán que unirse a tiempo para controlar la explotación de los yacimientos de carbón de manera parecida a como se controla la explotación de los bosques en estados bien organizados.» 
Es decir, no podemos saber (y no inporta cuál sea el volumen y el ritmo de la «acumulación de capital») si el fututo sezá más próspero que el presente. No existe, pues, ese motivo concreto para «descontar el fututo». Desde este punto de vista, la aplicación de la regla de Gray-Hotelling podría indicar que conviene no consumir ninguna cantidad de recutsos agotables ahora.

La situación es muy distinta a la que se plantea en la teoría (metafisica) del crecinniento económico, en la que se considera que un aumento de la inversión lleva a un aumento de la producción (por lo menos a largo plazo, es decir, prescindiendo de los ciclos causados por deficiencias temporales de demanda efectiva). En esa teoría sí que se supone que el futuro será más próspero que el presente y las tasas de interés sirven para igualar el valor actual, descontado, a las corrientes de ingresos a lo largo del tiempo.

Cabe sin embargo suponer que (aun en el caso de tasa de interés igual a cero o negativa) la regla de Gray-Hotelling determinaría una pauta de extracción óptima a lo largo del tiempo, si suponemos que los precios futuros en vez de subir, van a bajar. De hecho, no sabemos ni podemos saber cuáles van a ser las demandas en el futuro, y cualquier suposición al respecto tequiere adivinar la evolución de las técnicas y adjudicar un poder de compra, distribuido internamente de cierta manera, a todas las generaciones futuras, que de otra parte no sabemos cuántas serán. La «crematística» falla a la hora de determinar precios y cantidades extraídas de recursos agotables a lo largo del tiempo a menos que incluyamos «desde fuera» postulados éticos tales como elegir un númeto determinado de generaciones, o elegir una tasa de descuento del futuro.

Me parece que a los economistas "crematísticos» les debe molestar la idea de que el sistema de precios sea incapaz (a menos que se le proporcionen, «desde fuera», supuestos de cariz abiertamente ético) de determinar una pauta de explotación de recursos naturales agotables a lo largo del tiempo.

Pero tre parece también que un intento de explicación meramente ecológica de la reciente historia económica de la humanidad sería muy insuficiente (aunque pueda servir para entender la economía de un grupo bumano como los tsembaga).

\section{LAS AVANZADAS IDEAS DE POPPER-LYNKEUS}

La ecología es incapaz de explicar por qué, por ejemplo, la parte de la humanidad que vive de una manera que apenas transgrede los límites 
ecológicos, que utiliza el flujo de energía solar y no abusa ni del stock de combustibles fósiles ni de los materiales escasos y de reciclaje total imposible, es sin embargo la parte de la humanidad donde el hambre tiene mayor incidencia, mientras que la parte de la humanidad que, por así decir, menos respeta las leyes de la ecología lo pasa mucho mejor.

Sostener que la situación no puede continuar así y que habrá que igualar las oportunidades de vida fácil y agradable, tespetuosamente con la naturaleza, es un juicio ético (que yo comparto), pero to es una profecía científica. Que, por ejemplo, la «revolución verde» en la agricultura (que de «verde» en un sentido político no tenía nada, pues se basa precisamente en una agricultura petrolífera) no sea extendible al mundo en general (Pimentel, 1979), no implica que no pueda continuar casi indefinidamente en California.

La especie humana tiene la capacidad de comportarse como si de especies distintas se tratara; su comportamiento ecológico puede ser muy diversificado, tal vez mucho más de lo que puede suceder en otras especies. En la humanidad, con la densidad de población actual e incluso con densidades mucho más altas, tanto el hambre como el despilfarro son fenó menos sociales, que la ecología no puede explicar. Por otro lado, la capacidad de la humanidad paza comprender las leyes de la naturaleza puede influir, por ejemplo, en el control de población, de una manera muy distinta a lo que suceda en una especie animal.

Para sociedades humanas concretas, la ecología no puede predecir ni prescribir, más allá de la regla muy general de que no es posible transgiedir en todas partes y por mucho tiempo los límites ecológicos. Se trata, pues, de hacer una economía que no preste atención exclusiva a la determinación de precios y cantidades intercambiadas sino también, sobre todo, al uso y a la distribución de energía y de recursos materiales agotables. Se trata de una economía que no oculta su intención normativa de cara al futuro, ante la flagrante insuficiencia de la crematística para asignar recursos a lo largo del tiempo y ante la incapacidad de la ecología de prescribir, en detalle, para sociedades concretas, cómo debería ser ese uso y esa distribución.

Un ejemplo es la obra de Josef Popper-Lynkeus, Die allgemeine Näbrpflicbt («El deber general de nutrición como solución de la cuestión social... con una prueba de la falta de validez teórica y práctica de la teoría económica»). Popper fue físico y filósofo de la ciencia y su dedicacion a la economía llegó al final de su vida. Fue además literato, bajo el pseudónimo Lynkeus que añadió después a su apellido.

En ese libro de 1912 estudia minuciosamente la disponibilidad de energía y de recursos naturales en Alenania (aunque él era austríaco) y la posi- 
bilidad de proporcionar a todas las personas un mínimo de subsistencia. Ese mínimo sería proporcionado por el estado y existiría una especie de servicio laboral obligatorio para llegar a la consecución de ese mínimo universal. La producción para satisfacer las apetencias por encima de ese mínimo seguiría, por el contrario, las reglas de la libertad de mercado, y de cualquier modo no podría ser muy abundante. La última parte del libro estudia si ese sistema sería viable a lo largo del tiempo, suponiendo que las reservas de carbón ( $y$ otros recursos naturales) no puedan seguirse explotando con la misma intensidad. El problema «malthusiano» (como el propio Poppet lo llama) de recursos naturales y crecimiento de la población es discutido sin utilizar el lenguaje de los economistas, sino desde un punto de vista físico-ingenietil unido a una explícita postura ética. (Los in. genietos habitualmente utilizan los precios del mercado. Popper por el contratio escapó de la contaninación crematística.)

Es importante notar que Popper carecía absolutamente de cualquier intención social-darwinista. Al contrario, ataca abiertamente a Häckel y niega que su idea de eliminar la pobreza tenga nada que ver con la discusión contemporánea de un «mejoramiento de la raza» (Popper era judío y fue discriminado profesionalmente, como explica en su autobiografía). Su agradable falta de patriotismo alemán queda muy clara en su actitud favorable a un descenso de la población alemana, una propuesta valiente en vísperas de la guerra mundial.

Aunque no hay aquí espacio para hacer una recensión completa de la obra económica de Popper-Lynkeus (ni para estudiar su influencia en auto. res como Otto Neurath, teórico de la «economía de guerra» y participante del gobierno revolucionatio de Munich en 1919, que fue detribado con ayuda de la socialdemocracia alemana, destacado miembro del ala izquietda del Círculo de Viena, fundador de la «Enciclopedia de Ciencia Únifcada»), sí que es conveniente dar alguna muestra, resumiendo una parte, de particular resonancia actual, sobre las posibilidades de sustitución del carbón:

«Una aplicación parecida [a la de la madera] de la radiación solar indirecta en la forma de utilización de la vegetación que se renueva cada año sería producir, en gran escala, el alcohol que es ahora producido de las patatas. El alcohol puede proporcionar tanto luz como calor y fuerza, lo que no es pequeña ventaja, y aquí se trata otra vez de establecer las relaciones cuantitativas pertinentes.

"Según dice Krafft en Betriebslebre (p. 105), de un $\mathrm{kg}$ de patatas se puede obtener del 8,5 al 12 por ciento de alcohol en litros, es decir en promedio el 10 por ciento, es decir, supongamos un hectolitto de alcohol 
por cada mil $\mathrm{kg}$ de patatas. Ahora bien, de una hectárea de patatas se puede obtener (según el Calendario Agtícola de Mentzel y Lengetcke, p. 77, como también según el profesor Delbrück) de 100 a 240 quintales métricos, hasta 320 quintales como máximo, mientras que hoy la producción media es sólo de 130 quintales por hectárea. Calculemos que se obtenga la máxima producción de 320 quintales métricos por hectárea y considerando que el calor de combustión de un $\mathrm{kg}$ de alcohol es de 6.300 calorías (kcal, JMA), así por hectárea obtendríamos al año $32.000 \mathrm{~kg}$ de patatas, de las que obtendríamos $2.560 \mathrm{~kg}$ de alcohol, y de ese alcohol conseguiríamos unos 16 millones de calorías (...). Si ahora nos preguntamos si la calefacción doméstica podría hacerse con alcohol, en vez de con gas procedente de carbón, comprobamos que (...) haría falta (...) 8,44 millones de hectáreas de patatas (...).

»No hay que callar que muchos, por ejemplo Kropotkin, piensan que es posible aumentar veinte veces las cosechas de patatas por hectárea por encima de las actuales, con lo cual sólo haría falta 0,4 millones de hectá reas de campos de patatas para la calefacción doméstica de las viviendas de sesenta millones de personas en Alemania. En su obra La conquête du pain (p. 286 y ss.) Kropotkin dice que ya hay ejemplos de cultivos bajo invernaderos, en los que "las verduras se ponen debajo de vidrios $\mathrm{y}$, en tres meses, mediante esas simples coberturas de vidrio y una ligera calefacción se obtienen cosechas fabulosas, por ejemplo, $450 \mathrm{hl}$ de patatas por hectárea... hoy hacen falta veinte hectáreas para eso".

"Pero, en contra, hay que considerar el gasto de combustible para la calefacción de esas instalaciones. Kropotkin da un ejemplo de Jersey, donde son necesarias mil toneladas de carbón, para cuatto hectáreas de inver. naderos; de eso se sigue que para los 0,4 millones de hectáreas antes mencionadas, haría falta quemar cien millones de toneladas de carbón, imientras que el uso del alcohol serviría para ahorrat solamente setenta millones de toneladas de carbón para gas! (...)

„Una aplicación del alcohol para fines de transporte, o agricolas, o para iluminación podría ser más práctica. Los 3,3 millones de hectáreas de patatas actualmente existentes representan, suponiendo la máxima productividad, $3,3, .10^{6} \cdot 16 \cdot 10^{6}$ calorías (kcal, JMA) sin descontar el carbón para las destilerías; comparando con las 7.500 calorías (kcal) por $\mathrm{kg}$ que da el carbón de piedra, las calorías del alcohol setían equivalentes a siete miLlones de toneladas de carbón de piedra. Eso es algo, desde luego, y además tendría el efecto beneficioso de que no habría alcohol para beber, aunque tampoco quedaría disponible ninguna patata para comer.» 
En este texto vemos, pues, ya expresada muy claramente la idea de la competencia posible entre combustibles y alimentación que hoy, por ejemplo, pone de manifiesto el programa de fabricación de etanol de caña de azúcar (para automóviles) en Brasil. Popper-Lynkeus se hubiera negado a pensar que una tal elección debiera hacerse a través del sistema de precios. Vemos también una clara percepción de la idea del coste energético de producir energía: hace falta energía para calentar los invernaderos y para que funcionen las destilerías y también (aunque en el cálculo no se incluye) hacía ya falta también alguna energía de combustibles fósiles para producir los abonos para las patatas, etc.

Precisamente en esos años en Alemania se discutía con frecuencia qué cantidad de energía eléctrica haría falta para sustituir las muy cuantiosas importaciones de salitre de Chile -el proceso de tomat el nitrógeno del aire, iniciado en Noruega, requiere grandes cantidades de electricidad. Popper-Lynkeus hace al respecto un cálculo en su lỉbro, una señal más de su percepción clata de que la energía (ya sea en la forma de biomasa o en cualquier otra forma que no sea el calor solar que gratuitamente nos llega tiene un coste energético.

Ése es el tipo de consideraciones que puede llevar a una discusión razonable sobre la viabilidad de nuevas tecnologías y sobre las perspectivas económicas de la humanidad.

Tanto Kropotkin como Popper-Lynkeus eran fuertemente igualitaristas. El primeto creía mucho más que el segundo en el «progreso» material de 1a humanidad. Creía en un "comunismo libertario» en la abundancia futura, pero creía también, al mismo tiempo (a diferencia de la mayoritatia corrien. te dentro del marxismo) en la implantación inmediata de un «comunismo libertario en la escasez». Los postulados éticos de ambos son contrastados con discusiones sobre el "problema malthusiano», discusiones que en ambos casos no adoptan el lenguaje de los economistas: es decir, no se trata de alejarse de la realidad para discutir si la «acumulación de capital», el "progreso técnico», etc., podrán contrarrestar la tendencia de los «tendimientos decrecientes». Se trata de estudiar ecológicamente la economía (incluyendo, como advierte Popper, también los flujos de materiales) y de proponer soluciones que eliminen la pobreza. Lástima que Popper-Lynkeus no escribiera un libro sobre la economía mundial, en la misma perspectiva.

Su eurocentrismo se nota, por ejemplo, en la discusión que sigue a la del hipotético empleo del alcohol de patata. Popper escribe que, ya con el libro en pruebas, el ingeniero Rudolf Diesel le ha comunicado que sus motores pueden funcionat con aceites vegetales y concretamente con aceite de cacahuete. Sigue un cálculo sobre la eficiencia respectiva de máquinas de 
vapor que funcionan con carbón y de motores diesel, para establecer la equivalencia entre uno y otro combustible y para poder discutir la posibilidad de sustituir carbón (también petróleo) pot esa energía tenovable, siempre en la perspectiva de discutir la estabilidad a largo plazo de una economía que proporcione a todos el mínimo de subsistencia y que progresivamente vaya dejando de utilizar combustibles fósiles. A falta de algunos datos (por ejemplo, la cantidad de aceite de cacahuete que podría producirse por hectárea), Popper reserva su opinión, pero más bien se muestra favorablemente dispuesto a considerar que podría importarse aceite de cacahuete (que desde luego Alemania no puede producir) de las colonias alemanas en Africa y de España. La opinión, las necesidades, de los colonizados no son incluidas en la discusión.

Si Popper-Lynkeus hubiera adoptado un punto de vista mundial, sería hoy el más fuerte candidato a «santo patrón» del movimiento "verde» en Alemania (que hasta ahora lo desconoce).

\section{LA SEPARACION ENTRE LAS CIENCIAS}

El buscar tales antecedentes tiene interés en cuanto permite rastrear las razones y los efectos de la separación entre ciencias naturales y ciencias sociales. Algunos de los primeros críticos «ecologistas» de la ciencia económica (Geddes, por ejemplo) eran explícitamente comtianos y creían en una jerarquía de las ciencias - -el ser comtiano no le impedía, en casos como éste, decir cosas sensatas, ni implicaba tampoco que participara de las ideas políticas de «orden y progreso» de Comte. Otros críticos, sin adoptar esa posición epistemológica, creían que el estudio del uso de la energía ( $y$ de los tecursos naturales agotables) serviría para profundizar la discusión sobre teoría del valor económico (Podolinski), o para establecer límites o posibilidades de creciniento (Pfaundler). $\mathrm{Ni}$ todos esos autores eran pesimistas (la posición del propio Clausius es al final ambigua, pues hace un canto no cuantificado a la hidroelectricidad; Soddy hizo notar la potencia. lidad económica de la radioactividad ya en 1903, aunque no créa realmente en el crecimiento económico) ni todos eran igualitaristas (no ya frente al mundo en general sino incluso con refetencia a sus propios países). Uno de los subtítulos del esclatecedor artículo de Pfaundler es: «La lucha por la existencia es una lucha por energías libres.» Aunque Pfaundler (por lo menos en ese artículo) no hace el lazo entre análisis de la disponibilidad limitada de energía y darwinismo social dentro de la especie humana, y aunque no he leido aún ningún texto que desarrolle ese punto de vista, 
desde luego debe baber existido (algunas de las expresiones de Soddy, que era un hombre de izquierda, son ambiguas, incluso racistas).

Tal vez lo que les molestaba de la ciencia económica ortodoxa no era su apología del orden social y su peculiar énfasis en la «supervivencia de los más aptos (crematísticamente)», sino simplemente el carácter metafísico de algunos de los conceptos manejados por los economistas. Por eso son tan interesantes las críticas a Marx de autores situados claramente a la izquier$\mathrm{da}$, como Podolinski, o más tarde Popper-Lynkeus -implícitamente al menos, la crítica era sobre todo a la vaguedad de la noción de «fuerzas productivas». Quienes sabían hablar de «fuetzas» etan, por supuesto, los físicos.

A Marx se le puede reptochar que, tomando como objeto de conocimiento no «la economía» o «la sociología» sino la historia global de la humanidad, no entendiera a tiempo que las ciencias naturales también son ciencias históticas: eso es claramente así en el caso de la biología, pero también lo es en el caso de la física, de las leyes de la energía. Un buen libro de historia debe empezar con el Big Bang -en el siglo pasado, al menos, debería haber acabado y podría haber acabado a partir de la década de 1850 con una discusión sobre la hipótesis de la «muerte térmica» del universo. Que Jevons, por ejemplo, interesado en discutir con Maxwell las posibilidades de «reciclaje» de la energía, consiguiera separar tan radicalmente la historia natural del universo (y de la Tierra) de la ciencia económica (concebida como análoga a una mecánica —estática por tantode los deseos y satisfacciones humanas) le hace merecedor al puesto elevado que tiene entre los teóricos de la economía, despreocupados de la historia. Que Engels, por otro lado, que profesionalmente no era ni quetía ser «economista», ni «sociólogo», no biciera el esfuerzo de integrar los resultacos de las distintas ciencias en una única visión de la verdadera historia total (historia de la naturaleza e historia de la humanidad), es de lamentar, sobre todo porque su interés por las ciencias naturales y su despreocupación profesoral y profesional le pusieron en posición favorable, que no consiguió aprovechar del todo (a pesat del estímulo que le dio Podolinski).

Además, esa perspectiva integradora hubiera coincidido bien con el ptoyecto marxista de fututo. Así, en los últimos años, la discusión de las implicaciones políticas de la escasez de recursos naturales agotables ha tomado la dirección de vincular falta de crecimiento con distribución más igualitaria de los bienes disponibles. Muchos de los modernos economistas «ecologistas» son igualitaristas, tanto entre los científicos (Georgescu-Roegen, Daly, por ejemplo) como entre los más directamente políticos (Harich).

Se comprende que al abandonar las ilusiones de crecimiento continuado (e incluso las de un «estado estacionatio», como Georgescu-Roegen señala), 
se plantee de nuevo la cuestión de la distribución con toda su fuerza, tanto dentro de cada país como internacionalmente. Hay que hacer notar, sin embatgo, que no hay conexión necesaria entre la conciencia de la limitación del crecimiento y el igualitarismo. Una economía sin crecimiento, o en declive, no exige necesariamente una distribución más igualitaria. Aunque fallen algunas de las formas de legitimación de la desigualdad, como ha sido principalmente la confianza en el crecimiento futuro para todos, pueden nacer otras. Concretamente, en los estados europeos y en Norteamética, pueden darse las ptopias de una sociedad estamental y neocorporativa bajo gestión socialdemócrata, o puede darse el tenacimiento del darwinismo social bajo gestión política neoliberal. $\mathrm{Y}$, a falta de legitimación, puede haber violencia de una parte y miedo y resignación de la otra parte (dentro de cada estado y, desde luego, internacionalmente).

\section{LA CRITICA DE MAX WEBER AW. OSTWALD}

Tormenos a la cuestión de la separación entre las ciencias. El elogio de esta separación es la base del ataque de Max Weber, en 1909, a Wilhelm Ostwald, que había publicado ese año su libro Fundamentos energéticos de la ciencia de la cultura. Ostwald era en cierto modo un enemigo fácil porque, aunque decía algunas cosas sensatas sobre las ventajas que a los economistas reportatía conocer las leyes de la física, va mucho más allá, llevado por su fanático «monismo» reduccionista, y pensaba ser capaz de explicar todo tipo de fenómenos culturales (como el desarrollo del lenguaje). Lo quze particulatmente me interesa, en el contexto presente, es la desaprobación de Max Weber del «salto mortal» de Ostwald al campo de la economía.

Para entender esta crítica hace falta citar largamente a Max Weber. El fragmento que traduzco discute, al empezar, la idea de Ostwald (insatisfactoria por muchos motivos) de que el desarrollo de la «cultura» depende de la disponibilidad de energía (olvidando incluso la disponibilidad de materiales) y depende también de la eficiencia de la transformación de esa enetgía (ahí Weber compara telares mecánicos y manuales, los primeros con mayor coste energético por unidad de producto, aunque sin duda son, dice él, un «progreso técnico»). A este trecho, sigue la discusión que verdaderamente me interesa sobre la definición de «economía», que quizás no se entendería sin el anterior. A continuación, pues, este largo fragmento de Max Weber: 
"(Dice Ostwald que) "casi todo lo que sucede en la Tierra" sucede a costa de la energía libre que el sol da a la Tierra por la radiación (...). Una economía duradera debe por tanto basarse exclusivamente en el uso regular de la radiación solar anual, Ia eficiencia de cuya utilización es capaz todavía de un aumento tan enorme que el rápido gasto de la energía de radiación convertida y almacenada en energía química en las reservas de catbón (lo que sería una violación de aquel principio, algo parecido a la dilapidación de una herencia) no le parece nada grave. EI autor no habla del consumo de (...) hierro, que en comparación con las reservas existentes es sólo un poco más lento que el del carbón, ni tampoco de las reservas de cobre y de zinc que son tan importantes para la producción de electricidad, etc. En una exposición que incluso tiene en cuenta la edificación futura de nuestra economía energética sobre la energía de la radiación solar concentrada, filtrada y convertida en energía química o eléctrica, también hubiera sido seguramente oportuna una discusión de hasta qué punto (...) el aluminio, prácticamente inagotable y de rápida y continua disminución de costes, es hoy capaz de substituir las funciones indispensables de aquellos metales que indudablemente son agotables en la práctica.

¿Tanto más por cuanto Ostwald no cree en una disminución del abastecimiento de energía de la radiación solar en el pasado y en el futuro, dentro de las épocas geológicas, y por tanto aparentemente no parece nada urgente de cara al futuro y desde el punto de vista puramente energético el economizar en cierto grado las cantidades de energía que nos llegan desde allí, mientras (...) las materias que son indispensables para la producción, conducción y aprovechamiento de las energías útiles más importantes, son disipadas por el uso tan irremediablemente como sucede con todas las energías libres según la ley de la entropía. En este caso, sin embargo, a diferencia de otros, esto sucede en espacios de tiempo históricamente previsibles: si la explotación continúa al mismo titmo que ahora, en poco más de un milenio. Al atender exclusivamente la discusión a las relaciones energéticas -es decir: a) la obtención de nuevas energías primatias, $b$ ) el mejoramiento de la eficiencia en la obtención de energías útiles- no se discute entonces en absoluto el papel también importante de los conductores de energía que existen sólo, en gran parte, en reservas agotables, como objeto de la economía.

"Es decir, las cualidades que determinan la utilidad de esos conductores pueden incluirse con mucha dificultad y en todo caso sólo indirectamente bajo esas dos rúbricas, aunque no debe dudarse que la terminología de Ostwald también sea capaz de esto. Ahora bien, si para el futuro los aspectos de la utilización directa de nuevas energías son tan favorables como Ostwald supone tan confiadamente, especialmente la energía solat que hoy 
sólo es utilizable a través de las plantas vivas o fósiles, entonces se plantea la cuestión siguiente para el análisis energético de la cultura: zpor qué vamos a dar cualquier importancia a la eficiencia de las transformaciones energéticas, en esa situación, y teniendo en cuenta nuestras cifras de natalidad, que generalmente disminuyen? ¿Por qué no es entonces la eficiencia crecientemente irrelevante en vez de ser cada vez más importante? Una respuesta a esa cuestión sólo se podría eventualmente sacar con bastante esfuerzo y parcialmente de las exposiciones en el cap. IV ("La Vida"), V ("La Humanidad"), VI ("La dominación de las energías exteriores"). Si Ostwald la hubiera planteado y contestado expresamente, habría sido conducido a un análisis, de un modo sin duda útil a sus tesis, de unos problemas como los abordados por Sombart en su discusión del concepto de máquina de Reuleaux. Éstos son mencionados en la página 82 (parte inferior) rápida. mente y sobre todo de manera oblicua: no es correcto en modo alguno que el "progreso" de la cultura (cualquiera que sea el criterio corriente con que se mide el "progreso") sea idéntico a una disminución absoluta del uso de energía humana. Eso puede ser cietto para la importancia energética relativa de la energía humana al comparar la cultura contemporánea con, por ejemplo, la cultura antigua, pero no lo es ni tan siquiera en este sentido relativo para todo "progreso cultutal" - a menos que definamos como "progreso cultural" lo cue es "progreso" enetgético, lo que concluye en una tautología. Esas consideraciones omitidas quizás también hubieran beneficiado a Ostwald en su salto mortale al terreno de la disciplina específica de la economía (cap. XI). Se habría entonces evitado la proposición muy errónea, claramente deducible de sus exposiciones, de que por to menos eso que llamamos progreso técnico siempre descansa sobre una me. jora de la eficiencia. Como si, por ejemplo, al cambiar del telar a mano al telar a máquina, imputando la energía solat de radiación contenida en el carbón a las distintas energías cinéticas, químicas (extrahumanas y humanas) y otras, asignando la parte que corresponde a cada producto textil (incluyendo naturalmente la parte de energía no utilizada y disipada) y comparando esto con la cuesta correspondiente para el telat a mano, te. sultaría que la pura eficiencia energética fuera siempre tnejor en el funcionamiento mecátrico que con el trabajo manual.

"Los "costes" económicos distan mucho de correr simplemente en paralelo con el gasto de "enesgía" en el sentido físico de la palabra, y aún más se aleja, en la economía de intetcambio, la telación entre los precios de coste (que son los decisivos para la capacidad de concurrencia competitiva) de la relación entre las cantidades de energía utilizadas, aunque éstas influyan por supuesto en todas partes de manera muy "enérgica" (...). Si, como espera Ostwald, se tiene exito realmente en descubrir 
un aparato para convertir ditectamente la energía de los rayos solares en, por ejemplo, energía eléctrica, es posible que su eficiencia energética sea varias veces inferior a la de la utilización de la energía de carbón en una máquina de vapor y que, sin embargo, al mismo tiempo la capacidad de concurrencia competitiva de la energía obtenida de ese nuevo modo sea arrolladora. De hecho, la herramienta más «primitiva» dada al hombre por la naturaleza, el músculo humano, tiene una eficiencia mejor en la utilización de la energía que es liberada a través del proceso bioquímico de oxidación que la eficiencia que pueda alcanzar inciuso la mejor dinamo -y sin embargo, pierde en la comparación. Ostwald, sin duda, sabe perfectamente por qué. Pero en esta oportunidad Ostwald trata otra vez más de fundamentar "todo el desarrollo cultural" en simplemente uno de los distintos condicionamientos energéticos, es decir, en la eficiencia energética, aunque él mismo (ver más arriba) al principio presentó también la disponibilidad de nuevas energías. Incluso el puro problema tecnológico no es aftontado por Ostwald desde el punto de vista energético. Pues justo la te. lación opuesta entre la valorización de las nuevas energías y el mejoramien. to de la eficiencia sería lo propiamente interesante. Sobre esto, sin embargo, no se nos dice nada de importancia. No obstante, incluso la especificidad de una perspectiva como la económica (en sentido especializado), tan próxima a la tecrología, resultaría insuficiente para ello.

"Sin duda Ostwald ha hecho inicialmente la reserva de que es consciente de que tratará sólo un aspecto de los "fenómenos culturales", y esto es $\sin$ duda digno de reconocimiento frente a la necesidad de "fórmulas universales" de muchos pensadores naturalistas. Pero su mala estrella le hace creer todavía en la "jerarquía comtiana de las ciencias", ya tan cadu. ca, y que interpreta (p. 113, parte inferior) de manera que, como los conceptos de las ciencias "generales", que están en los escalones bajos de la pirámide, tienen validez para las ciencias colocadas más arriba, es decir, "menos generales", deben por tanto servir de "fundamentos" a éstas. Va a sacudir la cabeza, escéptico, si se le explica que para la teoría económica (esa parte específica de las disciplinas económicas, que las sepata de las demás), no sólo esos conceptos no tienen papel alguno, ni el más pequeño, sino que para la economía en general justamente los teoremas de las disciplinas "generales", los más abstractos y por tanto más aiejados de la experiencia cotidiana, carecen totalmente de importancia. Por ejemplo, que la astronomía acepte el sistema copernicano o ptolomeico le es totalmente iguai. Asimismo, para la validez de la teoría económica (esencialmente, unas proposiciones teóricas hipotéticas "ideal-típicas") sería totalmente irrelevante el que, por ejemplo, la teoría física de la energía experimentara los cambios más fundamentales, incluso si la ley de la conservación de la ener- 
gía continúa afirmando, como es de esperar, su campo de validez para todo conocimiento físico, qufmico o bioquínico o, si un día, un anti-Rubner le da la vuelta a sus experimentos sobre el gasto de calor en los organismos (lo que desde luego es sumamente improbable). $O$, para aclarar la cuestión con tespecto a los problemas que durante tanto tiempo han ligado la investigación física a los intereses económicos: incluso la existencia real de un perpetuum mobile, es decir, de una fuente de energía de la que brotara energía libre, sin coste, en un sistema energético dado, 1. No convertirf́a en «incorrecta» ni una tan sola de las proposiciones hipotéticas de la teoría económica abstracta, 2 . En el terteno de la validez práctica de esas proposiciones abstractas e hipotéticas, sólo sería reduciđa a cero, por colosales que se imagine uno, con toda la razón, las implicaciones de una tal utópica fuente de energía, si mediante esa fuente de energía estuviera disponible a) cualquier energía, $b$ ) en todo lugar, c) en todo momento, d) en cantidad ilimitada en cualquier período y $e$ ) con la dirección para el efecto deseado. Cualquier limitación por ligera que fuera de una sola de estas condiciones haría tomar inmediatamente al principio de utilidad marginal ura partícula de probabilidad, de significación práctica. Nos hemos entretenido en estas utopías sólo un momento, para dejar claro lo que tan frecuentemente se olvida en las modernas teorías sobre el método: que la jerarquía de las ciencias de Comte es un esquema de un pedante grandioso que ignota la realidad y no comprende que hay disciplinas con objetivos distintos de conocimiento, cada una de las cuales debe trabajar y sublimar el contenido del conocimiento no científico obtenido a partir de ciertas experiencias diarias directas, bajo puntos de vista distintos, totalmente autosuficientes. Que las distintas autodisciplinas se crucen luego en algún lugat y se encuentren, en sus objetivos, de manetas vatiadas, es evidente, y ocurre con la economía ya en su primer paso fuera del terreno de la teorfa "pura".»

\section{CIENCIAS SEPARADAS: CIENCIAS «APOLITICAS»}

Los precios, se supone, miden escaseces relativas para una mejor asignación de recursos escasos. Dependen de la demanda ahora y a lo largo del tiempo (es decir, de la intensidad y distribución del poder de compra ahora y a lo largo del tiempo), y depender de la oferta actual y futura (que mucho tiene que ver con las leyes de la naturaleza). Si los precios pueden o no pueden str tema de conocimiento aislado es una pregunta, a cierto nivel, trivial. Desde Aristóteles (posiblemente antes) 
está escrito que si un monopolista de sandalias acapara éstas, el precio sube: la crematística es un tipo de investigación con antigua tradición. De manera menos trivial habría que preguntarse si es posible hablar de la formación de precios sin estudiar las influencias sobre la oferta y la demanda presentes y futuras, influencias que son consideradas «extraeconómicas» arbitrariamente. Del lado de la demanda, la discusión sobre gustos, por ejemplo, se corta con la tautología del concepto de utilidad o con la simplificación de las «preferencias reveladas» (simplificación de patadójica aplicación cuando quienes las han de «revelar» aún to han nacido). Del lado de la oferta, nótese que Max Weber no sólo cree que da lo mismo si la «mecánica celeste» es ptolomeica o copernicana, sino que también da lo mismo si existe o no existe el segundo principio de la termodinámica: aunque hubiera un perpetumm mobile, con tal que hubiera un poco de escasez de algo en alguna parte, regiría el «valor» determinado por la utilidad marginal. Y como no hay, ni puede haber, perpetuum mobile, hay escasez, por tanto rige el «valor» determinado por la utilidad marginal. Peto, ¿cuánta escasez?, ¿de qué tipos de energia?, ¿de qué materiales? ¿Por qué la teoría económica construye sus «funciones de producción» con «tierra», «trabajo" y "capital», por qué supone que hay sustituibilidad entre ellos, cómo puede juzgarse esa clasificación (como si el capital, insistía Soddy, no se construyera con recursos naturales y energía) y cómo puede juzgarse ese supuesto básico de sustituibilidad?

Las dificultades de la teoría económica pura para determinar precios y cantidades «óptimos» a lo largo del tiempo han sido mencionadas: el predecir el futuro económico-crematístico está más allá de los poderes de la teoría económica «pura».

La competencia entre el "músculo humano» y la dinamo eléctrica depende a primeta vista de los precios relativos: pero esos precios encierran juicios éticos sobre el ritmo adecuado del gasto de combustibles fósiles.

Que la economía se cruce con otras disciplinas ya en su primer paso fuera de la teoría económica «pura» significa, presumiblemente, que para hacet historia económica no puede usarse sólo teoría económica «puta», sino que hay que tener en cuenta, por ejemplo, en una expresión cara a los economistas, el «marco institucional».

Ahora bien, consideremos algunas explicaciones históricas típicas de economista profesional. Kuznets, por ejemplo, da series de las magnitudes macroeconómicas a lo largo del tiempo, uexplicandon el camino distinto de distintas economías por el porcentaje de la «inversión» dentro del ingreso total. ¿Explica esto lo que está sucediendo en los últimos años, con un aumento considerable en todas partes de la relación incremental capital/ producto? Es decir, hay cada año «inversión neta» (es decir, aumento de 
la «capacidad productiva») y sin embargo el «producto» apenas crece, incluso disminuye. iY para salit de esta situación, los economistas en todas partes predican un aumento de la «inversión»! ¿No oculta, precisamente, esa terminología los fenómenos reales que se habian dado y que se están dando? En ciencias naturales difícilmente se permite a una teoría "pura» que realice tan malas predicciones. ¿No se debe un tal fracaso al uso de categotías metafísicas como «inversión», «producción» (sin especificar, por ejemplo, si la «invetsión» consiste en fábricas para «producir» aluminio o en nuevas irrigaciones de tierras secas)?

Otro ejemplo: en cualquier texto de historia económica se puede leer que a medida que aumenta la "productividad» de la agricultura (lo que ocurre debido al «progreso técnico» que sustituye trabajo y tierra por ca" pital), la agricultura libera trabajadores y puede liberar tecursos que ayudan al desarrollo de otros sectores (con tal que, por ejemplo, la telación de precios sea adecuada, lo que seguramente es probable que ocurra, debido al carácter competitivo de los mercados agrícolas, con muchos vendedores, transfriéndose los aumentos de «productividad» a una baja de precios te. lativos). Cuando en estos otros sectores la «productividad» aumenta, no necesariamente disminuye, de momento, el empleo en ellos, pero eso sí que ocurre en la agricultura debido a la débil elasticidad-ingteso de la demanda de productos agrarios en general. La aplicación de conceptos de teoría económica a la historia económica da un tipo de explicación (y, en este caso, ha sustentado durante largos años unas tecomendaciones a los países subdesarrollados) que, a la luz de la física y de la biología, aparecen como muy dudosos ya que la productividad energética de una agticultura petro. lífera es inferior at de una agricultura tradicional.

Es decir, para hacer historia económica de verdad, no basta con añadir a la teoxía econónica «pura» el «matco institucional» (y un poco de eco. nometría retrospectiva) sino que haría falta, primero, aclatarse sobte la adecuación a la realidad de conceptos como "productividad», «producción», «sustitución de trabajo y tierra por capital», etc.

Que hay objetos de conocimiento diferentes puede aceptarse (por ejemplo, la formación de los precios en un sistema de mercado es ciertamente un «objeto de conocimiento» separable del estudio del sistema de gobierno en esa sociedad: no se puede estudiar y escribir a la vez sobre el equilibrio del monopolista y sobre las formas de legitimidad o ilegitimidad de ese gobierno). Que esos objetos de conocimiento pueden después integrarse en un estudio histórico es obvio en la misma práctica de Max Weber.

Más allá de Ia sospecha de que muchas personas persiguen cátedras (especializadas) más que conocimientos, más allá de la manifiesta dificultad de ser competente en todas las ciencias, hay que encontrar ottas tazones que 
expliquen la separación ( $\sin$ síntesis postetior de resultados) de la física y de la economía, a pesar de los esfuerzos pioneros de unos pocos autores (cuyos textos es posible presentar como relativamente novedosos, tanto para los físicos como para los economistas, lo que demuestra que la separación ha durado hasta hoy: por ejemplo, los físicos se sorprenden cuando leen al Clausius economista; los economistas no saben quién era Clausius).

Pero cualesquiera que sean esas tazones, el becho de que esos antecedentes integradores existan es importante. La crítica «ecológica» de la cien. cia económica es muy anterior a 1973, es incluso anterior a GeorgescuRoegen y a Boulding.

Posiblemente, la falta de integración hay que buscarla en la pretensión de «apoliticismo» de las ciencias. Cuantos más «objetos de conocimiento» distintos se integren en una visión histórica cada vez más global, tanto más nos vemos capacitados para decir qué ha ocurrido en el mundo y para establecer una cierta congruencia entre lo que ha venido ocurriendo y lo que puede, y deseamos, que ocurra. Se supone que los científicos no desean que ocurra nada en particular. Como lo que ocurte en el mundo nunca es ni exclusivamente físico, ni químico, ni biológico, ni económico-crematístico, ni demográfico, sino una mezcla de todo ello, cuanto más especializado sea el conocimiento menos se puede decir globaimente, lo que tiene la virtud de poder pasar desapercibido, sin tener que dar la cara política.

Así, por ejemplo, el economista profesional puede construir modelos sobre el uso de tecursos agotables a lo latgo del tiempo, haciendo las hipótesis que tenga por conveniente sobre el desarrollo o falta de desarrollo de nuevas técnicas. Retrospectivamente, un historiador que quisiera explicar el uso real de recursos agotables sin tener en cuenta los cambios técnicos reales que se han dado, no sería aceptado - la «contrafactualidad» no puede llevarse tan lejos. En cambio, al economista se le permite en la profesión decir algo, que supuestamente informa sobre la probabilidad de distintas posibilidades en el futuro, sin justificar, en el terreno de las ciencias correspondientes, sus hipótesis sobre futuras tecnologías. Introduce, por ejemplo, una back-stop technology tal que, a determinado nivel de precios, empieza a resultar competitiva: si se le pregunta cuál es concretamente, te puede contestar legítimamente dentro de las reglas de la profesión que no es asunto de su incumbencia, sino de los departamentos de la Facultad de Ciencias. Si el presente y el futuro se estudiatan y proyectaran como historia, y como historia (verdaderamente) global, eso no podría ocurrit. 


\section{REFERENCIAS COMENT ADAS}

Los textos de Clausius, Popper-Lynkeus y Max Weber proceden de:

Rudolf Clausits, Ueber die Energievorräthe der Natur und ibre Verwerth. ung zum Nutzen der Menschbeit, Verlag von Max Cohen \& Sohn, Bonn, 1885 (debo este texto a Klaus Schlüpmann).

Josef Poppet-Lynkeus (1838-1921), Die allgemeine Nährpflicht als Lösung der sozialen Frage, Carl Reissner, Dresden, 1912 (el texto traducido está en las págs. 729-30).

La autobiografía de Popper se titula Mein Leben und Wirken (Carl Reissner, Dresden, 1924). En inglés, hay una biografía en Paul Edwards (ed.), Encyclopedia of Pbilosopby (Macmillan) — donde también hay una biografía de Otto Neurath. Schumpeter no les menciona en su historia de la ciencia económica.

Max Weber, «Energetische Kulturtheorien», Arcbiv für Sozialwissenscbaft und Sozialpolitik, 29, 1909, también en Gesammelte Aufsätze zur Wissenschaftslebre (3." edn., hrsg. Johannes Winckelmann), J. C. Moht (Paul Siebeck), Tübingen, 1968.

El libro de W. Ostwald se titula Energetische Grundlagen der Kulturwissenscbaft, Alfred Kröner, Jeipzig, 1909. Ostwald (que era químico, gran empresario académico, premio Nobel) tiene otros libros en la misma línea, El imperativo energético, Filosofía de los valores. Del libro citado creo que existe una versión francesa.

El texto de Max Weber alude a Franz Reuleaux (1829-1905), director de Ia Technische Hochschule de Berlin, autor de Theoretische Kinematik (1875), y a Max Rubner (1854-1932), sucesor de Koch en Berlín, quien determinó exactamente el uso de energía en la nutrición animal y humana (Die Gesetze der Energieverbrauchs bei der Ernäbrung, 1902).

El criterio de Gray-Hotelling está explicado en libros tecientes de teoría económica de los recursos agotables, por ejemplo Anthony C. Fisher, 
«Papers»: Revista de Sociologia

Resource and Environmental Economics, Cambridge U.P., 1981. L. C. Gray publicó su artículo «Rent under the Assumption of Exhaustibility» en el Quarterly Journal of Economics, 28, 1914, y H. Hotelling, «The Economics of Exhaustible Resources» en Journal of Political Economy, 39, 1931. Una discusión excelente sobre la cuestión del «descuento del futuro» se encuentra, aparte de las obras de Georgescu-Roegen, en los comentarios finales de H. Daly, en H. Daly y A. Umaña, Energy, Economics, and the Environment, AAAS Symposium, Westview Press, Boulder, Colorado, 1981.

Sobre Patrick Geddes (1854-1932) he comentado algo en mi artículo en Mientras Tanto (Batcelona), 12, 1982. Aparte de Cities in Evolution (1915) Geddes publicó ya en los años 1880 unos folletos: An Analysis of the Principles of Economics (Williams and Norgate, Edimburgo, 1885), The Classification of Statistics and its Results (A. \& C. Black, Edimburgo, 1881), Jobn Ruskin, Economist (1884, hay una ed. reciente). Hay por lo menos dos libros sobre la vida de Geddes, de Philip Mairet y Philip Boardman. Una crítica de Geddes a la teoría económica está en una carta suya a Walras de 1883 , publicada en la correspondencia de Walras.

Sobre Serhii Podolynsky (1850-1891), J. M. Naredo y yo hemos publicado un artículo cuya última versión es «A Marxist Precursor of Energy Economics: Podolinski», en Journal of Peasant Studies, 1982. Klaus Schlüpmann ha escrito una biografía que se publicará seguramente en 1983 en el JPS. Podolynski no era tan desconocido como pensábamos, hay artículos y libros sobre él publicados en Ucrania, y en Montreal se prepara una edición completa de sus escritos. Su artículo (discutido por Engels) que, me parece, es el primer intento de «economía energética», fue publicado inicialmente a Revue socialiste (Lyon), 1880, y luego en italiano, ruso y alemán.

El artículo de L. Pfaundler (n. 1839) (otro físico austríaco) se titula «Die Weltwirtschaft im Lichte der Physik», Deutsche Revue, 27 (2). Stuttgart y Leipzig, 1902. Es un artículo muy claro, que propone sin embargo la dudosa idea de que los materiales pueden siempre reciclarse, si se dispone de bastante energía (el «dogma energético», como le llama GeorgescuRoegen).

Anterior a Pfaundler, es el libro de E. Sacher (otro austríaco), Grundzüge einer Mechanik der Gesellschaft, Gustav Fischer, Jena, 1881, que incluye una contabilidad energética de inputs y outputs en la agricultura. Sacher publicó otro libro parecido en 1899.

Sobre Frederick Soddy, véase el artículo de H. Daly en History of Political Economy (1980). EI subtitulo de su folleto Cartesian Economics (1922) es muy explícito: la influencia de la ciencia física sobre la administración del estado. La biografía de Soddy en el Dictionary of Scientific 
La ciencia económica y el análisis energético

Biograpby (ed. pot Gillespie) no se toma en serio a Soddy como economista: ¿Como es posible que un Nobel de química se ponga, en su madurez, a escribir destempladamente contra los economistas y predique la superio tidad de Ruskin como economista?

El interés de Jevons por la energética no aparece sólo en The Coal Question (1865) sino también en su correspondencia posterior (publicada por Macmillan, Londres, con Maxwell, Herschel y otros científicos); aparece además en su libto sobre la lógica de la ciencia. Peto está ausente, claro está, en sus "principios de economía» marginalista, donde el modelo es la mecánica.

Otros libros citados son:

D. y M. Pimentel, Food, Energy and Society, Arnold, Londres, 1979.

W. Harich, Comunismo sin crecimiento, Materiales, Barcelona, 1978 (?).

Por último, agradezco la simpática ayuda de Sabine Hecker para la traducción del texto de Max Weber. 